Voix et Images

\title{
Maria Chapdelaine au Canada anglais : réflexions sur notre extravagance
}

\section{François Paré}

Volume 2, numéro 2, décembre 1976

Paul Chamberland

URI : https://id.erudit.org/iderudit/200058ar

DOI : https://doi.org/10.7202/200058ar

Aller au sommaire du numéro

Éditeur(s)

Les Presses de l'Université du Québec

\section{ISSN}

0318-9201 (imprimé)

1705-933X (numérique)

Découvrir la revue

Citer cet article

Paré, F. (1976). Maria Chapdelaine au Canada anglais : réflexions sur notre extravagance. Voix et Images, 2(2), 265-278. https://doi.org/10.7202/200058ar d'utilisation que vous pouvez consulter en ligne.

https://apropos.erudit.org/fr/usagers/politique-dutilisation/ 


\section{Maria Chapdelaine au Canada anglais: réflexions sur notre extravagance}

Le texte qui suit résulte d'un travail de recherches conçu en 1973 , dans lequel nous nous étions proposé d'évaluer l'accueil réservé à Maria Chapdelaine dans la presse et dans les revues canadiennes-anglaises depuis la parution du roman jusqu'à nos jours. Notre étude était d'emblée descriptive. Mais, une fois rédigée, vu l'importance de dépasser les limites autarciques que le Québec s'est fixées depuis toujours quant à ses œurres d'art, notre description a pris aussi bien la forme d'une démystification. II aura fallu, pour un temps, renoncer à l'idée d'une collectivité suffisante, espérant voir dans cette discrétion de la méthode une manière détournée de confirmer par ailleurs notre collusion et notre indissociabilité.

Sans doute parce qu'elle s'attachait à dépeindre une configuration du pays québécois, l'œuvre de Louis Hémon a largement débordé les cadres du Québec. Le roman, on le sait, a connu de très nombreuses traductions, autant d'éditions étrangères, autant de lecteurs "sédentaires" soudainement immergés dans «l'atavisme lointain du vagabondage». De ces outrances où Maria Chapdelaine s'est trouvée en quelque sorte dépaysée, nous n'avons retenu que la plus absolue, la plus infime et, par conséquent, la plus définitoire de toutes: la canadienne-anglaise.

Ce projet nous engage alors sur un étrange parcours où, en dehors de nous-mêmes pour quelques instants, nous allons nous regarder par les yeux de l'Autre, nous délinéer dans son regard même comme en un prisme. La critique canadienne-anglaise nous fournira un angle insolite (insolent?), une sorte de route tangentielle, au bout de laquelle, si nous avons accompli le détour nécessaire, le mythe de Maria ne sera plus qu'une histoire et une magie trompeuses. Le livre a connu une telle renommée à l'intérieur de notre discours culturel que le Canada ne pouvait que réagir: c'est un peu l'histoire de cette réaction qui nous

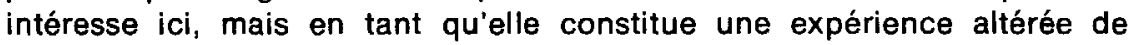
nous-mêmes, contexte de l'étranger à la fois aveugle et délibéré qui nous a contraints à l'existence.

Notre analyse se fera en deux temps. D'abord, nous tâcherons de rendre compte aussi explicitement que possible du dépouillement que nous 
avons fait du «dossier canadien-anglais ${ }^{1}$ ». En deuxième lieu, et plus généralement, nous énoncerons les quelques intuitions que nous aura léguées l'étude de l'ensemble de ces textes sur la co-existence historique des deux cultures au Canada.

C'est au gré d'une méthode assez impressionniste que nous décrirons maintenant quelques-uns des articles consacrés à Maria Chapdelaine, selon ce que l'on pourrait appeler après coup (nous verrons comment) une thématique de la dévalorisation.

Au Canada anglais, ceux qui ont parlé de ce roman sont fort peu nombreux. Dans l'ensemble, l'Angleterre, à elle seule, a davantage remarqué l'œuvre de Louis Hémon que ne l'a fait le Canada d'expression anglaise en cinquante ans ${ }^{2}$. Par exemple, dans le Winnipeg Free Press, on ne retrace qu'un seul article sur Louis Hémon, pour la période allant de 1923 à 1973. Dans le Globe and Mail de Toronto: aucun article. Dans le Ottawa Journal: un seul. Deux essais uniquement ont été publiés par la Revue de l'Université de Toronto pour la même période. Il n'est évidemment pas question du Vancouver Sun, ni du Halifax Herald ${ }^{3}$. Le corpus compte à peu près vingt-cinq articles: environ dix de ces articles ont paru dans les pages du Montreal Star et commémorent l'ouverture du musée Louis Hémon à Péribonka.

L'ensemble des critiques canadiens-anglais a cru comprendre le roman dans ce qu'il atteignait de plus secret et de plus universel. Les textes de ces hommes, souvent d'illustres inconnus, donnent lieu à une matière très diverse: articles de journaux, articles polémiques, contreparties de discours électoraux, rubriques publicitaires, traductions, anthologies, etc. C'est aussi morcelé que se présente ce miroir où nous avons voulu nous regarder de l'extérieur.

Cette écriture, bien qu'elle paraisse considérable, s'échelonne sur de nombreuses années et elle est entourée d'une immense pudeur à parler du roman. II faut bien voir que les quelques vingt-cinq textes repérés ne constituent que le fractionnement passager de ce qui n'a été, au fond, qu'une tenace réticence au pouvoir de la parole.

Au départ, le Canada anglais n'a jamais contesté, comme cela aura été le cas aux États-Unis, le statut d' «immortel chef-d'œuvre» que la francophonie en général avait conféré à Maria Chapdelaine ${ }^{4}$. Au contraire, les critiques d'Andrew MacPhail et même celles d'érudits plus contemporains ont fait de la timide Maria l'héroïne d'une tragédie dont elle-même n'aurait pu présager l'existence. Livrées aux «Anglais", les voix qu'entendait Maria n'étaient plus que des signes obliques, et le roman; où Louvigny, de Montigny avait lu un appel du pays et Lomer Gouin une "épopée nationale", 
était devenu un texte éminemment poreux, ouvert et friable sur tous ses côtés.

Délaissant les facettes périlleuses d'un roman auquel le Canada français attachait, à ses yeux, trop d'importance, la presse canadienneanglaise a concentré son intérêt sur la biographie de Louis Hémon. On invente sans arrêt, dans toutes les directions; on cherche des fondements scientifiques à une vie qui les a ignorés tous. L'édition canadienne du Times publie un important reportage sur Éva Bouchard, "The Peribonka Belle who claims to be original of Maria Chapdelaine". De son côté, C. McNaught, dans un numéro du Canadian Magazine de 1935, raconte une sorte de pèlerinage romancé au pays des bleuets. L'auteur de l'article se substitue étrangement aux personnages de Louis Hémon: il rêve, il devient le Québécois moyen cueillant les bleuets sacrés, il entend, lui aussi, les voix du Nord. II se reconnaît enfin dans ce paysage saturé de mots dont l'abord et la compréhension, dit-il, lui ont toujours été refusés. C'est un voyage dantesque. L'article de McNaught constitue un vibrant hommage à tout ce que n'est pas l'œuvre de Hémon.

Le long travail de Madame Audrey Freeman, présenté à la Sorbonne en 1951, n'échappe pas à la séduction de la biographie. Les grands titres de la thèse révèlent assez clairement les intentions du critique: "Vie et ceuvre de Louis Hémon avant son séjour au Canada, Louis Hémon au Canada, Commencement d'ennui à Londres, Soif de voyages lointains ", etc. Audrey Freeman découvre essentiellement une homologie entre la vie de l'écrivain, son désir d'aventures et de nouveauté, et la trame de ses œuvres de la Belle que voilà jusqu'à Maria Chapdelaine. "Voit-on maintenant le tempérament de l'homme, la manière de l'écrivain 5 ?"

Il est intéressant de noter, du reste, avec quelle passion les commentateurs canadiens-anglais se sont consacrés au mystère de la'mort de Louis Hémon. Cet attachement est étrange en apparence seulement, car le jeune écrivain, qui avait tant aimé l'Angleterre (dit-on), n'était-il pas disparu au moment où il s'engageait sur la "bonne" voie? Ne marchait-il pas vers l'Ouest justement, vers ce qu'était le Canada du futur ${ }^{6}$ ? "He could have been Canadian ", s'exclame C. McNaught.

Canadien? Oui, Louis Hémon aurait pu l'être, si l'on avait pu éliminer la malencontreuse erreur que constituait la rédaction de Maria Chapdelaine.

La critique d'expression anglaise se consacre donc, en général, à l'étude de l'inoffensif. Elle n'atteint jamais la substance fictive du livre, ni ses répercussions dans la collectivité québécoise. Eile s'est concentrée surtout sur l'écrivain lui-même, lequel, si ce n'est dans son œurre, au moins dans la quotidienneté, s'est passionné pour le Canada.

Ainsi, dans le Winnipeg Free Press, un certain W.F. Osborne relate les événements tragiques de Chapleau. Le journaliste explique le succès de Maria Chapdelaine au pays non seulement par la qualité du récit et la 
vraisemblance des personnages, mais aussi et surtout par le «pathos d'une mort prématurée". Ailleurs, dans un article intitulé "Beloved Vagabond's grave», le Père S. MacManus fait l'éloge de l'aventurier d'origine française et du poète de tradition très chrétienne.

Les rubriques publicitaires reprennent à bon compte l'éloge inconditionnel que l'on peut retrouver dans la presse internationale vers 1925. Ces entrefilets insistent sans doute moins sur le caractère moralisant et chrétien du livre de Hémon, car ils veulent désigner des lieux communs plus enracinés dans la tradition britannique. De ce fait, même si elle ne possède pas l'ampleur des cuvres de Dickens, la publicité range volontiers Maria Chapdelaine parmi les grandes épopées du monde moderne. Le Montreal Star parle maintes fois d'une “charmante romance " dotée de qualités héroïques.

Il est évident que l'attirail publicitaire canadien-anglais, renonçant à des cordes trop nationalistes, a conçu son éventuel public lecteur comme instruit dans l'héritage littéraire issu du monde victorien. II y avait là, dans cette publicité, une représentation d'elle-même que jamais la collectivité québécoise (et ses élites) n'aurait pu soupçonner. Notre apanage culturel et les liens ambigus que nous entretenions avec la France, tout cela subissait un transfert total. Nous étions évidemment victoriens; et ce roman nous représentait, quelques bribes enlevées à Dickens.

Encore ne s'agit-il que de la publicité «officielle», celle défrayée par les éditeurs. En 1922, le Ottawa Journal, dans un compte rendu de Maria Chapdelaine, relie le roman aux périodes les plus obscures de la colonisation du Québec. L'histoire de Maria met en scène, non plus des individus "réels", mais des types d'individus, ceux-là mêmes qui habitaient ce que le journaliste appelle les «backwoods" du Québec. (Le journal, pourtant publié si près de Montréal, semble croire que la totalité du territoire québécois est constituée de "backwoods"; ou encore, ce qui est pour le moins subtil, que cette rusticité sert de critère en vue de déterminer le type du Québécois.) Enfin, en dépit d'une déficience dans l'usage de la langue, ajoute le quotidien de la capitale, le Canadien français, représenté par Samuel Chapdelaine, est aussi un «enfant de la Nature", comme l'avait peut-être été dans la mère-patrie le paysan irlandais.

De là, il n'y a qu'un pas à faire de Maria Chapdelaine une héroïne typiquement européenne. La mince critique anglo-saxonne au Canada s'est toujours empressée de "dévaloriser" le roman, non dans ses qualités narratives (ce qui paraissait particulièrement inintéressant), mais dans ses implications socio-politiques, en accentuant les aspects autobiographiques de l'œuvre, comme nous l'avons vu, et en décentrant les lieux où devait se jouer l'action des personnages. On faisait ainsi sauter les cordes du "vrai" et de l'intraduisible que notre élite québécoise aimait brandir. Maria Chapdelaine met en scène, soit des cas particuliers choisis parmi la petite société du Lac Saint-Jean, soit une typologie universelle. Ce qui est rejeté par la critique canadienne-anglaise, c'est ni plus ni moins le discours cul- 
turel qui, au Québec, faisait de ce roman une représentation privilégiée de la collectivité. Plusieurs textes, notamment dans les revues universitaires, s'acharnent inconsciemment à dépouiller Maria Chạpdelaine de son enracinement dans l'histoire idéologique du Québec, en rappelant que l'auteur était bel et bien un voyageur français et en enfonçant les personnages dans une tradition de type britannique ou européen.

A ce titre, le Pr Philippe Grove, de la Société royale du Canada, publie en 1944 une étude de Maria Chapdelaine dont les objectifs sont fort sérieux. Pour l'homme de lettres torontois, le roman, dont on ne compte plus les exemplaires vendus, se trouve à la croisée de deux mondes. La trame, dans laquelle l'héroïne de Hémon est peu à peu emprisonnée, prend sa source dans le conflit insoluble de deux forces typiquement coloniales: d'une part, une instance de changement, purement dramatique; d'autre part, un élément de permanence, purement épique. Maria, face au changement absolu, décide finalement de rester sur la terre paternelle. Maria Chapdelaine, au dire de Grove, met en cause l' «unité tribale des Canadiens français " en tant qu'elle se rapporte aux désirs contradictoires d'une indépendance accrue de la colonie et d'une métropole française puissante. Ainsi, le roman, bien que situé au Québec, pourrait constituer une cuvre universelle, ou, au moins, une tragédie occidentale. Tragédie d'une transhumance enlaçant des cultures faites, depuis toujours, pour l'isolement et la solitude.

Un certain B.K. Sandwell, dans le Canadian Home Journal (1939), s'aventure plus loin que $P$. Grove et manipule justement des concepts que le Canada anglais a toujours voulu éviter dans ses relations avec le Québec. A son avis, il faut accorder beaucoup de mérite à l'œuvre de Louis Hémon, car la publication du roman marque un événement à caractère national. "Maria Chapdelaine is a great contribution to the unity, the mutual understanding of the two great divisions of the Canadian people." La parution de Maria Chapdelaine, avance encore l'auteur de l'article, constitue un fait "Canadian", car, pour la première fois, une œuvre nordaméricaine échappe au caractère "défensif» de la voix coloniale. En ce sens, conclut Sandwell, écrit par un étranger de passage, possédant donc l'altérité nécessaire, le roman omet les deux divisions de la nation canadienne et transforme l'esprit défaitiste de la colonisation en enthousiasme et en unité.

On ne peut douter de la sincérité de certains articles. Celui du Canadian Bookman de 1921, bien que bref, contient un monceau de documentation. On y retrouve une étude comparée des deux traductions anglaises, avec des textes parallèles à l'appui. L'auteur de cet article anonyme décrit en particulier le nombre effarant d'éditions qui ont marqué la vie du livre dès les premiers moments de sa parution. Canadian Bookman encourage les maisons d'enseignement canadiennes à inscrire Maria Chapdelaine au programme des lectures obligatoires. Cette cuvre, en effet, en. plus de posséder une valeur morale évidente, peut instruire adéquatement la jeunesse d'expression anglaise sur les mœurs et les coutumes de leurs 
voisins immédiats. " $A$ work of the most poignant simplicity and beauty", Maria Chapdelaine sera donc consacré au maintien impérieux de l'unité canadienne.

Canadian Bookman se dit assuré que Louis Hémon ignorait tout du clivage culturel dans lequel le Canada d'alors se trouvait partagé; il ne pouvait porter attention à des conflits qui, somme toute, s'estompaient devant "l'action immémoriale de la Poésie". "Louis Hémon writes as if he did not know (and we are very sure that he did not care) that there was in Canada any such thing as a 'clash' or a bilingual question, or Imperialism,... ${ }^{7} \gg$ II est intéressant de voir combien une telle ignorance de la part de Hémon est souhaitée par le Canada anglais, pour qui la reconnaissance même d'une présence française est l'acceptation d'une incurable maladie (et une condition de survie) ${ }^{8}$.

Dans un article extrêmement documenté (Maclean, 1953), Margaret Zieman entreprend, à son tour, une sorte d'archéologie du séjour du voyageur français chez les Bouchard de Péribonka. Les entrevues qu'elle mène auprès de Samuel Bédard, d'Éva Bouchard («The Peribonka Belle") et des compagnons de Hémon conduisent $M$. Zieman à faire de l'écrivain un mésadapté typique de l'Europe impériale du début du xxe siècle. II aimait voyager, dérivant d'un pays à l'autre, selon les caprices et les emplois du moment. Poursuivant son commentaire, Margaret Zieman s'attache désespérément à repérer des structures de pensée dont les éléments individuels, une fois rassemblés, conféreraient au roman un réalisme rassurant. Eliminer le mythe qui entoure l'œuvre, cela veut dire essentiellement gratter la surface de la narration jusqu'au modèle inopérant qui lui a donné naissance et qui, à la limite, forme la seule vérité sur laquelle il vaille la peine de s'appuyer. Il fallait décaper le plus possible l'invention et le romanesque, ne laisser là comme preuve que le récit réel, la Maria Bouchard, trop timide pour proférer quelque revendication que ce soit. Pour des raisons divergentes, toutes deux au nom du "vrai", les élites québécoises et canadiennes, on le pressent, ont dû radier la plupart des aspects imaginaires du roman pour se consacrer à la vie de son auteur. Simultanément, c'était le réalisme qui, en 1925, suscitait et reniait la vision d'une authenticité québécoise.

La presse canadienne-anglaise, de 1920 à 1925, démunit presque entièrement l'œuvre de Louis Hémon de sa "valeur" et de son emprise idéologique. Le roman n'est plus que la trame d'une intrigue amoureuse; les personnages pourraient être de tous les climats et de toutes les latitudes. Non seulement Louis Hémon est-il le cas caractéristique du vagabond européen, mais ses personnages sont aussi des modèles d'universalité. Samuel Chapdelaine, par exemple, devient le symbole de la lutte accablante (struggle) que mène la paysannerie mondiale afin de prendre en charge sa destinée. Qu'aurait dit Louvigny de Montigny s'il avait appris que le «chef-d'œuvre» dont il vantait les mérites était de facture communiste? 
Au dire de plusieurs, Maria Chapdelaine s'inscrit assurément dans le voisinage immédiat des Géorgiques de Virgile, des Pêcheurs d'lslande de Loti, du Vicaire de Wakefield de Goldsmith, etc. Les personnages du roman de Louis Hémon possèdent encore un courage "spartiate"; ils accomplissent des tâches "herculéennes"; ils évoquent une catholicité dont l'abbé Prévost aurait été envieux; enfin, les revendications de Maria ressemblent à celles mises de l'avant par la jeunesse londonienne.

De même que la découverte d'une origine biographique (chez McNaught et Zieman) était rassurante (par la soi-disant véracité du roman), l'adjectivation que nous venons de citer joue un rôle similaire, en dépouillant l'œuvre de ses particularités locales. Faire de Maria Chapdelaine un "classique» avant l'heure, c'était maintenir une sorte d'indemnité, vite naturalisée, autour d'un mythe qui présentait beaucoup de danger. Au sens le plus primordial, cela consistait donc à retirer le roman du pouvoir politique et idéologique dans lequel il s'insérait forcément comme écriture et comme lecture. Pour la critique canadienne-anglaise, Maria Chapdelaine était une histoire vécue. Une seule interprétation invalidait toutes les autres. Le Canada anglais a voulu exclure le fourmillement oratoire qui accompagnait l'œuvre, de sorte qu'il n'y ait pas de texte "réellement " littéraire, “just a simple story », une vérité enfin, un reportage.

Y a-t-il eu des voix à Péribonka? La critique canadienne-anglaise s'est attachée à en retracer les témoins et à en adoucir la force politique. Elle a eu peur de l'invention; elle a craint que ses voix ne soient avant tout celles du texte même, voix périlleuses, s'il en est, car elles prolifèrent sans raison et sans repère.

Curieusement, ou encore par son essence propre, la traduction n'échappe pas à ce nivellement des valeurs et des idéologies. Dans un travail à maints égards fort intéressant, Jacques Bierre compare et évalue les traductions de Blake et de MacPhail 9. II procède par images et par motifs privilégiés, c'est-à-dire par ce qu'il croit être en émergence dans le système stylistique de Hémon. Cette méthode est précieuse car elle s'attaque très précisément à l'intraduisible. Les résultats de l'étude démontrent clairement que, dans ses deux traductions anglaises, Maria Chapdelaine s'est trouvée en partie neutralisée. Les symboles les plus essentiels s'y repèrent à peine; des expressions jugées outrancières sont rendues par des termes appauvris. Un mot comme "cri», précise J. Bierre, est souvent traduit par "sound", le mot «poigne" par "grasp", "exaltation» par "emotion», etc. A vouloir trop dire le roman, il semble bien que les traductions aient partiellement trahi l'intention de Hémon. Ce qui aurait paru sûrement au lecteur anglo-saxon comme de fâcheux écarts de vocabulaire a dû être dompté par une traduction plus pudique.

Alors que la grande majorité des lecteurs canadiens-français s'attachait à la valeur "poétique» et connotative de l'œuvre, l'élite añglophone, elle, s'employait à repérer l'endroit de la dénotation, là où les 
choses se sont passées, brèves, rassurantes et définitives. C'est ainsi que le Montreal Gazette décrit Maria Chapdelaine comme un «compte rendu émouvant [vivid] de la vie des Canadiens français du Nord du Québec". On peut conclure que l'effort de scientificité et le rigorisme qui caractérisent les textes canadiens-anglais à cette époque correspondaient justement à une angoisse devant le caractère polyvalent, dangereux et instable de l'œuvre à promouvoir.

Cela ne veut pas dire que Maria Chapdelaine soit restée inconnue des lecteurs canadiens d'expression anglaise. Au contraire, sous forme d'éditions successives chez Macmillan, ou encore disséminé dans des anthologies à l'usage des étudiants de langue, le roman est resté présent dans l'héritage scolaire de la plupart des Canadiens. Le roman, oui, mais l'idéologie véhiculée, elle, n'a jamais franchi la frontière du Québec. Par les yeux de l'Autre, nous étions profondément désincarnés. Privés de notre effervescence, privés de nos inventions, de notre langage meme, le Canada anglais nous engageait à survivre dans le roman d'un Français de France. Au reste, doublement éloignés de notre salut, ni Français, ni Anglais.

L'ensemble des articles dépouillés indique donc que, lorsqu'elle s'est fait entendre, l'élite canadienne-anglaise s'est représenté le roman soit comme une autobiographie, neutralisant du même coup le mythe du pays québécois, soit comme une tragédie universelle dont les personnages, curieusement, se trouvaient vivre dans la forêt du Nord canadien.

Par une sorte d'entente tacite (et certainement inexprimable à l'époque), la critique canadienne-anglaise a presque évité de parler d'un livre où sa parole même, en tant qu'instance collective, se laissait séduire et capturer totalement. II fallait parler de ce livre, mais sans en parler vraiment, en décentrant son rayonnement idéologique et en ramenant son extravagance à la timidité intime de la biographie. Maria Chapdelaine avait pour prénom Éva et pour créateur un homme qui aimait l'Angleterre; elle habitait un village tranquille du Nord québécois. Dans l'ensemble du pays, on peut dire que le "héros» Hémon a occupé une place prédominante; quant aux voix du texte, celles que Maria a entendues malgré tout, celles que nos porte-parole soulignent encore (comme le verra habilement Félix-Antoine Savard ${ }^{10}$ ), ces voix, le Canada les a ignorées, tout occupé qu'il était à expérimenter les choses, sa propre mort, son unité, l'Ouest, le Klondyke.

En tout une vingtaine de textes, publiés dans presque autant de périodiques différents, voilà la somme de ceux qui ont osé commenter l'œuvre de Louis Hémon. Le reste, si considérable soit-il, semble ne se laisser désigner que par le silence. 
Comment donc expliquer ce repli? Comment donc expliquer ce qui apparaît, en premier lieu, comme une abdication de la critique canadienne-anglaise devant le péril d'un roman qui se vendait par tirages exorbitants? S'agit-il d'une simple ignorance? Ou s'agit-il, au contraire, d'une stratégie?

Devant l'ampleur que prenait Maria Chapdelaine dans le monde francophone, il semble bien que les journalistes et universitaires canadiens aient privilégié une forme d'abstention où le problème de la "vérité" était résolument écarté, et où, subtilement, une critique se faisait avec efficacité sans pour autant émerger au grand jour.

Comment ce presque silence, donc, est-il devenu en vingt ans la réponse concertée du Canada anglais au mythe de Maria Chapdelaine? II est malaisé d'établir avec rigueur ce qui, en fait et par définition, n'a jamais été exprimé ouvertement. Pourtant, trois types de phénomènes peuvent se présenter à l'esprit.

En premier lieu, nous l'avons assez dit, il fallait supprimer un mythe dangereux et aplanir ce qui se révélait être, au Québec, une idéologie «nationale» et une entreprise d'identification collective. Il existait deux manières d'arriver à ces fins. D'une part, la critique canadienne-anglaise pouvait s'affirmer autre que celle qu'elle voulait récuser et ainsi instaurer une sorte de concurrence absolue. Or, n'était-ce pas là s'inscrire par contraste dans l'identité culturelle du Québec? Cette solution était désavantageuse, en effet, car elle soulignait et prolongeait un conflit qu'elle voulait bien voir se résorber au plus tôt. C'était très précisément nourrir la dualité, celle même qui, au Québec, alimentait les discours populaires. Le Canada anglais a toujours refusé cet affrontement direct et a préféré intégrer la collectivité québécoise selon le modèle familier de l'unité dans la diversité.

A l'opposé d'une confrontation radicale se trouvait le silence. Ignorés et laissés à nous-mêmes, nous étions sans échos et sans images. Par ses prémisses et par son indifférence, le silence obturait entièrement le mythe. On n'en parlait plus. Se taire, c'était confiner l'émergence d'un discours identificateur au Québec à l'intérieur de ses propres limites; c'était empêcher toutes les réverbérations par lesquelles notre collectivité se définissait. II n'est pas étonnant que, durant les nombreuses années de succès de Maria Chapdelaine jusqu'à ce jour, les journaux du Canada aient presque totalement omis une œuvre qui bouleversait l'opinion publique québécoise. Ce refus soutenu de la parole devenait le centre ouvert et tacite d'une critique du mythe identificateur au Québec, tel que manifesté chez Louis Hémon lui-même et chez les orateurs francophones. Par un silence à peine rompu (vingt-cinq textes), la presse canadienne-anglaise. pouvait sans contredit désamorcer le mythe; elle anéantissait le roman dans ses assises les plus primitives, comme événement social et comme publication. 
On peut évoquer d'autres cảuses à cette «ignorance» de la critique canadienne-anglaise. II suffit de mettre en lumière fort brièvement les conditions sociales par lesquelles les deux groupes ethniques se définissaient au début $d u x x^{\oplus}$ siècle: territoire très compartimenté, en particulier sur lîle de Montréal, intérêts culturels et politiques tout à fait divergents (comme en témoigne la fameuse crise de la conscription), discours et idéologies opposés, etc. On sait qu'en 1917, par exemple la motion Francoeur avait, en pratique, proposé l'indépendance du Québec. Au cours des débats, les parlementaires s'étaient même servis du roman de Louis Hémon, afin de ramener au bercail les députés dissidents ${ }^{11}$. II y a donc une sorte d'abstention légendaire, quasi «littéraire», invoquée entre les deux peuples, et l'intransigeance des élites ne peut que souligner cet écart. Peur d'une contagion culturelle trop facile, cette indifférence a sans doute modulé la réaction de la critique canadienne au succès de Maria Chapdelaine, et elle rappelle du même coup le désintérêt réciproque qui a affligé le Canada bilingue dès ses premiers moments.

Enfin, et troisièmement, les deux cultures marquaient, en 1920, une différence d'enthousiasmes. Les regards du Canada anglais s'étaient portés peu à peu sur des nouveautés et des exotismes que jamais le Québécois moyen n'avait contemplés. Tandis qu'au Québec le gouvernement plongeait dans l'entreprise désespérée d'une colonisation des terres du Nord, le Canada, lui, dirigeait ses désirs vers les plaines inexploitées de l'Ouest, posant peu à peu les bases de ce qui allait devenir un mythe et un trésor ${ }^{12}$. C'est donc l'Ouest de l'or et de la prairie qui séduit l'imagination des Canadiens, déjà affectés par les premières dégringolades de la dépression économique. Canadiens français et anglais étaient en quelque sorte incompatibles, non peut-être dans les motivations profondes qui les poussaient à se déplacer, mais dans les espaces géographiques au cœur desquels ils inscrivaient et répartissaient leurs espoirs.

Le désintérêt de la critique canadienne-anglaise à l'égard de Maria Chapdelaine n'était donc pas sans fondement réel. Pour le Québec, c'était le Nord mythologique dont Gilles Vigneault s'est fait le troubadour contemporain ( «le Nord du Nord»), celui de la Baie James. Pour le Canada: le transcontinental, le long voyage horizontal, la ligne propre de l'horizon. Aux yeux des Canadiens, la «mouvance" de Samuel Chapdelaine manquait irrémédiablement la "vraie" vie, l'aventure pragmatique où l'on défile sur une terre d'avance toute donnée! ${ }^{13}$.

En regard de l'incroyable vigueur du mythe de Maria Chapdelaine au Québec ${ }^{14}$, l'attitude des journaux canadiens-anglais n'a pu que dénoter le souhait d'une dévalorisation implicite de l'œuvre et de ses retombées. Elle trahissait, du reste, une répartition «naturelle" des tâches, imposée dès les premières années du régime colonial britannique. Laissant à ceux qui en manifestaient le désir l'entière liberté de parole, les dirigeants anglais ont pris en charge la pratique des choses ${ }^{15}$. L'élite anglo-saxonne n'a certes pas reconnu (ou voulu reconnaitre?) la séduction des "voix" du pays. Elle a joué la stratégie du silence, se gardant justement en marge du 
piège de la parole, n'y entrant jamais totalement, absente et présente à la fois, refusant le cercle du dire afin de prendre en charge l'économie hiérarchique des choses et les choses elles-mêmes.

A l'encontre des habitants du «pays de Québec " qu'ils considéraient de si loin et forcément, les Canadiens de 1920 constituaient une race qui avāit toujours su mourir. Et c'était normal. La vie au Canada, si l'on en croit les ouï-dire de l'histoire, est demeurée longtemps axée sur les désirs de la métropole anglaise. Ne venait-on pas de dépenser labeur, soldats et armes pour une guerre trop lointaine (la guerre des Boers)? La véritable indépendance, non pas celle du Statut de Westminster (1931), mais l'autonomie profonde, n'occupait pas les pensées de tous les jours, ni les politiques à long terme du gouvernement fédéral.

En plus d'une indépendance chancelante, le Canada de 1920 connaissait la démence d'une immigration déversant sur un territoire à peine occupé des milliers d'étrangers qu'il s'agissait de rendre canadiens. Pour cela, il aurait fallu pouvoir les loger au cœur d'une tradition nationale sûre, et il devient évident que l'idéologie véhiculée par Maria Chapdelaine ne pouvait satisfaire cet espoir. De 1920 à 1930, le Canada s'est montré préoccupé par le problème de son identité: apparition de la Banque du Canada, puis de la radio nationale, signature du Traité de Westminster, etc. C'est d'une sorte de continuité abstraite, éminemment morcelée, que le Canada semble être fait. Ses porte-parole sont moins unifiés qu'au Québec: ils ont leurs différences et les affirment. La politique pancanadienne rend toujours l'image d'un pays césuré et parcellaire tendant à son unité. Ce n'est nullement la survivance d'un peuple ici en jeu, mais l'engendrement de cette race “Canadian", périphérique et émiettée jusque dans son centre.

En 1920, nombre de Canadiens ne peuvent se représenter le pays idéal que sous forme de dépendance coloniale ${ }^{16}$. Pour eux, malgré la singularité de ses politiques, le Canada est une sorte de Grande-Bretagne déplacée. Il est la mère-patrie, et à la fois ne saurait l'être. C'est que la nation canadienne ne surgit toujours qu'au terme d'un renoncement à elle-même, d'une mort de soi par laquelle l'autonomie perdue dans ce sevrage est recouvrée ensuite plus forte et plus authentique. En vingt ans, sous les coups d'une immigration forcenée, le Canada anglais est devenu la victime de son manque d'identité. Décentré, hors de lui-même, en quête d'une origine fictive, il a l'impression de se voir, anglais à nouveau, déferlant sur le continent européen, avec le pied de l'Empire comme unité de mesure.

Par là, le Canada d'expression anglaise invoquait une forme privilégiée de représentation de soi, dans laquelle l'essence de la nationalité canadienne se modelait à l'extérieur d'elle-même, dans une distance si nécessaire qu'en elle la colonie se trouvait constamment réaffirmée. La culture canadienne, autour de la publication de Maria Chapdelaine, était devenue très exactement cet espace, ce miroitement de la mort, l'image révulsée de la métropole anglaise. 
A l'inverse, si la culture québécoise s'était montrée si inhospitalière jusqu'à Maria Chapdelaine, c'est qu'elle avait toujours omis l'hospitalité de la mort (ou encore du mort, en pensant, bien sûr, à Saint-Denys Garneau). Afin d'exister, il lui aurait fallu s'anéantir dans l'œuvre qui la faisait naître et, dès lors, jouer le jeu jusqu'à la fin.

Mais ce jeu trop mortel, on le sait, notre élite l'a bientôt refusé. C'est ainsi qu'au Québec les lecteurs de Maria Chapdelaine ont opté pour ce que Louvigny de Montigny avait appelé le «vrai». Et le «vrai » résidait dans la conformité aveugle de la fiction aux valeurs immobiles et non fictives du passé. La vérité ne faisait pas nécessairement l'objet d'une quête collective, comme dans la société post-révolutionnaire américaine. Elle devait être, au contraire, un enracinement et une durée. L'âge d'or devenait une réalité quotidienne inspirée par la fidélité, tandis que le présent (la présence même des choses) demeurait une redoutable fiction.

L'couvre tentée par l'habitant Chapdelaine restituait une origine à chaque fois intacte, comme si «faire de la terre» n'était pas déjà un acte de mort ${ }^{17}$. Chaque personnage se cantonnait soigneusement dans l'habitacle chaud de la répétition, là où l'imaginaire (les rêves de Maria) pénétrait sous forme de vie silencieuse et de fausse origine.

En un moment déconcertant, dans le noyau de I'histoire, le Québec s'est séparé de ce qu'il croyait être ${ }^{18}$. Événement important que celui-là, puisqu'en lui une culture a pu se dédoubler parfaitement. La tâche de l'œuvre littéraire, fixée par les autorités de l'époque, était justement de maintenir comme telles l'authenticité et la nostalgie d'un passé créé de toutes pièces. En voulant déterminer l'art comme non-artifice, le Québec rêvait d'une patrie hors-texte, alors que, sans le savoir, seul le texte que les poètes écrivaient à mesure savait conférer quelque existence à cette patrie. Longtemps, et encore aujourd'hui, le rôle de l'œuvre littéraire au Québec aura été de transformer la nostalgie en désir collectif. Cette littérature, que nous prétendions issue de Louis Hémon, repoussait avec désespoir le renversement capital de l'équilibre, l'instance coloniale, l'œuvre subite de la mort. Elle refusait l'événement même du Québec qui est une disparition et en même temps un récit.

Pendant les cinquante ans qui nous séparent de la première parution de Maria Chapdelaine, l'œuvre de Louis Hémon est passée, chez nous, au crible d'une démystification difficile. Malgré cela, il semble bien que nous soyions restés entre nous, parmi les nôtres, pesant le poids abrupt du réveil. Pendant ce temps, le Canada anglais, comme en témoigne encore Edmund Wilson ${ }^{19}$, suivait avec une sorte de stupeur, avec grand flegme, l'arpentage effaré de ce lieu au milieu de nous, la "lisière " que Hémon a si bien compris être le vide et l'absence pure.

Le Canada confédéral s'est produit "at large" comme une contradiction de faits: d'un côté, la terre de Maria Chapdelaine, ensemencée d'échecs, mais peuplée de convoitises par une nation sans mort, nation survivante, le Québec; de l'autre, c'est la mort continuelle, le Canada de la 
reine se masquant lui-même pour que la figure canadienne jaillisse dans son autorité. Le Canadien anglais, à l'encontre de Samuel Chapdelaine, accepte d'être second dans les rouages d'un Empire qui le soutient. II sait qu'il arrive trop tard. II sait qu'il a raté la chance américaine.

Au Québec, un sentiment bien différent s'est implanté peu à peu, et l'idéologie du terroir n'aura qu'à s'y infiltrer subrepticement. En perdant le soutien de la métropole française, le paysan québécois perdait aussi son statut de secondarité. Il lui fallait trouver sa définition ailleurs. Et ce lieu ne pouvait être qu'en lui-même. L'isolement dans lequel ont été plongés tous les Chapdelaine des $X V \|^{e}$ et $x \mid x^{\ominus}$ siècles avait servi à déterminer le territoire québécois comme une question de fait, une surface aux frontières reconnues et défendables depuis l'époque de la Conquête. En souhaitant un retour de la flotte française, le Québec aurait encore rêvé d'une origine fausse. Le "pays de Québec ", celui où Maria choisit de rester, était essentiellement constitué d'un manque. La métropole ne pouvait revenir en force sans pour autant nous détruire comme peuple. Rêve d'un absent maudit et irrécupérable dans la mesure où, retrouvé, il n'aurait été qu'une aliénation absolue de nous-mêmes.

Autour de nous, il n'y a donc eu que silence et différence. Dans ce bref survol du "dossier canadien-anglais", nous avons tenté de voir comment et pourquoi, étant si tapageurs au milieu de nous-mêmes, nous n'avions guère été entendus qu'avec crainte et réserve. Tout s'est passé comme si, en ces temps de discours, de monuments, de paroisses nouvelles, de pré-duplessisme, en ce temps des tout premiers mythes, nous avions rayonné d'effroi. Et ce détour dans l'histoire de la critique canadienne-anglaise aura surtout été décevant. Non, nous n'y étions pas présents. Personne, en fait, ne se trouvait là. II n'y avait d'extravagant que notre propre discours.

Par les yeux de cet Autre où nous avons voulu passer pendant quelques instants, on ne pouvait voir que le défaut de nous-mêmes. II n'y avait personne ou presque pour reconnaître notre désir collectif, personne d'autre que nous-mêmes pour entendre nos discours endiablés. Entourés d'une pondération toute anglaise, nous n'étions alors qu'une déraisonnable cacophonie.

A l'inverse, le Canada anglais, dans ses rapports avec l'idéologie québécoise, a toujours souffert de son propre dé-paysement. On peut se demander, comme nous l'avons vu, s'il n'a pas aussi souhaité et cu:tivé cet échec. 
1. Grâce aux travaux de Nicole Deschamps et de Nicole Hurtubise, une grande part des articles ici recensés peut être consultée à même les "dossiers Maria Chapdelaine ", au département d'Études françaises de l'Université de Montréal.

2. A titre d'illustration, le Times de Londres a fait paraître sept articles d'importance sur Maria Chapdelaine et sur son auteur dans les seules années 19211922. Les journaux anglais se sont intéressés grandement à cet ouvrage si populaire provenant de la colonie.

3. Selon les données fournies par chacun de ces quotidiens.

4. Le public américain a semblé, en effet, plus éveillé: «a perfect story in perfect French about only half-sympathetic French Canada " (New York Times, 22 février 1922).

5. Audrey Freeman, le Canada de Louis Hémon et sa destinée litteraire, Sorbonne, thèse inédite, p. 66 .

6. On sait que Louis Hémon a perdu la vie à Chapleau, alors qu'il marchait avec un compagnon sur les rails du chemin de fer transcontinental.

7. Canadian Bookman, décembre 1921, p. 7.

8. Cette problématique ne rappelle-t-elle pas l'interprétation pan-canadienne du célèbre mot du Général de Gaulle lors de sa visite de 1967?

9. Les traductions de Maria Chapdelaine, texte inédit, Université de Montréal, 1968. Ce travail peut être consulté à même le «dossier Maria Chapdelaine" ci-haut mentionné.

10. «Non! Être libre, c'était, partout où l'on va dans le pays, sentir, sous ses pieds, le son de la terre répondre aux battements de son cœur, c'était entendre partout la voix du sol, des bois, des eaux dire: "J'appartiens à ta race et je t'attends!" (Menaud maître-draveur, Montréal, Fides, 1937, p. 177).

11. Voir Louis Dupire, "Autour de la motion Francoeur", le Devoir, 23 janvier 1918, p. 1 et 5 .

12. Vers 1900 , Clifford Sifton, alors ministre de I'Intérieur du Canada, avait déclenché une vaste campagne en faveur de l'immigration. II avait pour projet de peupler la seconde moitié du pays. Les convois du Canadien Pacifique ont transportẹ vers l'Ouest des milliers d'immigrants. De 1918 à 1930, on calcule que plus de 1400000 d'entre eux se sont ainsi installés en terre canadienne. (Voir Rosaire Morin, I'Immigration au Canada, Montréal, L'Action nationale, 1966.) «ll s'agissait à la fois de développer l'Ouest, de noyer l'élément canadien-français, de fournir de la clientele aux réseaux et de créer une surabondance de main-d'œuvre évitant la hausse des salaires." (Robert Rumilly, Histoire de la province de Québec, XI, Montréal, Fides, p. 167).

13. Voir Michel Brunet, Histoire du Canada par les textes, Montréal, Fides, 1963, II, p. 31.

14. II faut consulter l'excellente thèse de Normand Villeneuve, Histoire d'une identification collective: le mythe de Maria Chapdelaine au Québec, Université de Montréal, 1971.

15. C'est l'Église qui maintenait les limites acceptables de la parole, et non pas le gouvernement colonial anglais.

16. Voir Michel Brunet, Histoire du Canada par les textes, II, p. 92.

17. Cet idéal, la Terre paternelle de Patrice Lacombe (1846) l'avait déjà renversé; mais le livre, malheureusement, était passé inaperçu. La mort du père y était comme transigée dans les choses mêmes et la terre, c'est-à-dire l'enracinement, y faisait l'objet d'un contrat.

18. Voir Fernand Dumont, le Lieu de l'homme, Montréal, HMH, 1968. "La conscience se trouve prise dans les jeux de la culture, se découvrant dans la distance qu'elle instaure et dans la pressante nostalgie de la réconciliation qui l'anime par ailleurs." (p. 11)

19. Voir Edmund Wilson, "Reporter at large", The New Yorker, vol. 40, 1964, p. 94. 